Submitted to Physical Review B

FSU-SCRI-98-40

CU-NPL-1159

\title{
Perturbation Theory for Spin Ladders Using Angular-Momentum Coupled Bases
}

\author{
J. Piekarewicz \\ Supercomputer Computations Research Institute, \\ Florida State University, Tallahassee, FL 32306 \\ J.R. Shepard \\ Department of Physics, \\ University of Colorado, Boulder, CO 80309
}

(October 8, 2018)

\begin{abstract}
We compute bulk properties of Heisenberg spin-1/2 ladders using RayleighSchrödinger perturbation theory in the rung and plaquette bases. We formulate a method to extract high-order perturbative coefficients in the bulk limit from solutions for relatively small finite clusters. For example, a perturbative calculation for an isotropic $2 \times 12$ ladder yields an eleventh-order estimate of the ground-state energy per site that is within $0.02 \%$ of the density-matrixrenormalization-group (DMRG) value. Moreover, the method also enables a reliable estimate of the radius of convergence of the perturbative expansion. We find that for the rung basis the radius of convergence is $\lambda_{c} \simeq 0.8$, with $\lambda$ defining the ratio between the coupling along the chain relative to the coupling across the chain. In contrast, for the plaquette basis we estimate a radius of convergence of $\lambda_{c} \simeq 1.25$. Thus, we conclude that the plaquette basis offers the only currently available perturbative approach which can provide a reliable treatment of the physically interesting case of isotropic $(\lambda=1)$ spin ladders. We illustrate our methods by computing perturbative coefficients for the ground-state energy per site, the gap, and the one-magnon dispersion relation.
\end{abstract}

\section{INTRODUCTION}

Heisenberg spin ladders have been extensively studied via a number of powerful theoretical methods including direct diagonalization [1], quantum Monte Carlo [1] and density matrix renormalization group (DMRG) [2]. Such investigations have been complemented by 
development of simpler, often analytic, treatments whose role is to help elucidate the physics underlying the numerical results. These approaches are typically based on perturbation theory and almost invariably assume a "dimer" or "rung" basis (see Fig. 1). Note that in the rung basis, the two-leg-ladder Hamiltonian becomes diagonal in the limit in which the coupling along the chain $\left(J_{\|}\right)$becomes negligible relative to the coupling across the chain $\left(J_{\perp}\right)$. In this basis, the intra-chain couplings are, thus, treated perturbatively. Such perturbative expansions are routinely assumed to be meaningful, even in the region where the inter-chain and intra-chain couplings are comparable (i.e., $J_{\|} / J_{\perp} \sim 1$ ) [1.3, 四.

In the present work we present a perturbative treatment of two-leg spin ladders implemented using a "plaquette" basis which, as we have argued elsewhere [0,6], is particularly well-suited to the study of such systems for a variety of reasons. Our method exploits the simplicity of these quasi one-dimensional systems to straightforwardly extract perturbative information about the bulk system from ordinary Rayleigh-Schrödinger perturbation theory. In so doing we avoid the more general, and, thus necessarily more complicated, diagrammatic methods of "connected graph expansions" [7]. Specifically we can, with modest computational effort, determine the bulk values of the ground state energy per site, the spin gap, and the one magnon dispersion relation to eleventh, fourth and third order, respectively. Moreover, by performing similar calculations in the rung basis, we can make reliable quantitative comparisons of the radii of convergence of the perturbative expansion in the two bases. Indeed, we determine that the radius of convergence in the rung basis is $\lambda_{c} \equiv J_{\|} / J_{\perp} \simeq 0.8$ while for the plaquette basis, where, as will be discussed below, $\lambda$ scales the inter-plaquette coupling, we find $\lambda_{c} \simeq 1.25$ to 1.4 . Clearly, the plaquette basis is strongly preferred for the treatment of the physically interesting [8] case of isotropic (i.e., $\lambda=1$ ) spin ladders.

Our paper is organized as follows. In Sec. II we briefly describe the rung and plaquette bases and outline how to apply Rayleigh-Schrödinger perturbation theory to finite spin ladders in these bases. In Sec. [II] our method for extracting perturbative information for bulk systems from the finite-ladder results is outlined. Our numerical results appear in Sec. IV where we also address the issue of the radii of convergence in the rung and plaquette bases. The paper concludes with a summary section.

\section{PERTURBATION THEORY IN COUPLED BASES: FINITE SYSTEMS}

\section{A. The Rung Basis}

For a two-leg ladder, the dynamics are described by the Heisenberg Hamiltonian with nearest-neighbor antiferromagnetic coupling:

$$
H=J_{\|} \sum_{\leftrightarrow} \mathbf{S}_{i} \cdot \mathbf{S}_{j}+J_{\perp} \sum_{\uparrow} \mathbf{S}_{i} \cdot \mathbf{S}_{j},
$$

where $J_{\perp}\left(J_{\|}\right)$is the strength of the antiferromagnetic coupling along the rungs(chains) of the ladder [1]. In the strong-coupling limit $\left(J_{\|} / J_{\perp} \simeq 0\right)$ rungs interact very weakly with each other and the above separation of the Hamiltonian is a natural one. Indeed, by fixing the large scale in the problem to be one $\left(J_{\perp} \equiv 1\right)$ the Hamiltonian can be separated into

the sum of $H_{0}$, which contains the intra-rung interactions, and a potential $V$-scaled by the 
small parameter $\lambda \equiv J_{\|} / J_{\perp}$, responsible for inducing interactions between rungs. In this way, the dominant intra-rung Hamiltonian can be written as (see Fig. 而)

$$
H_{0}=\sum_{r=1}^{N_{R}} h_{0}^{(r)} ; \quad h_{0}^{(r)}=\mathbf{S}_{1}^{(r)} \cdot \mathbf{S}_{2}^{(r)},
$$

while the inter-rung potential $(\lambda V)$ is expressible in terms of

$$
V=\sum_{r=1}^{N_{R}}\left[\mathbf{S}_{1}^{(r)} \cdot \mathbf{S}_{1}^{(r+1)}+\mathbf{S}_{2}^{(r)} \cdot \mathbf{S}_{2}^{(r+1)}\right] .
$$

Note that $r$ labels a specific rung and the sum runs over all $N_{R}$ rungs in the ladder.

The rung basis is particularly suitable in the strong-coupling regime, as $H_{0}$ is diagonal in this basis. Indeed, eigenstates(eigenenergies) of $H_{0}$ are a direct product(sum) of single-rung states(energies) of the form:

$$
\left|\phi_{\ell m}\right\rangle=\left|\left(s_{1} s_{2}\right) \ell m\right\rangle ; \quad \epsilon_{\ell}=\frac{1}{2} \ell(\ell+1)-3 / 4 .
$$

Here the two spins along the rung are coupled to a total angular momentum $\ell$, which can be zero or one, and projection $m$. What remains to be done is to evaluate matrix elements of the potential in the rung basis. To do so, we rely on the same angular-momentum techniques [9,10 that were used to evaluate matrix elements of the Hamiltonian in the plaquette basis [5].6]. We obtain

$$
\begin{aligned}
& \left\langle\ell^{\prime(1)} \ell^{\prime(2)} j m|V(1,2)| \ell^{(1)} \ell^{(2)} j m\right\rangle= \pm 3 \widehat{\ell^{(1)}} \widehat{\ell^{(2)}} \widehat{\ell^{\prime(1)}} \widehat{\ell^{\prime(2)}} \\
& \left\{\begin{array}{lll}
\ell^{(1)} & \ell^{\prime(1)} & 1 \\
\ell^{\prime(2)} & \ell^{(2)} & j
\end{array}\right\}\left\{\begin{array}{lll}
\ell^{(1)} & \ell^{\prime(1)} & 1 \\
1 / 2 & 1 / 2 & 1 / 2
\end{array}\right\}\left\{\begin{array}{ccc}
\ell^{(2)} & \ell^{\prime(2)} & 1 \\
1 / 2 & 1 / 2 & 1 / 2
\end{array}\right\} .
\end{aligned}
$$

Note that we have defined $\hat{x} \equiv \sqrt{2 x+1}$ and that the $+/-$ sign in the above expression should be adopted whenever $\ell^{(1)}+\ell^{\prime(1)}+j$ is even/odd. Moreover, the above expression vanishes whenever $\ell^{(1)}+\ell^{(2)}+\ell^{\prime(1)}+\ell^{\prime(2)}$ is odd. Aside from simple phases and numerical factors, the matrix elements of $V(1,2)$ depend on scalar functions (i.e., independent of $m$ ) known as Racah coefficients; here we cast the matrix elements in terms of the more symmetric $6-j$ symbols [9, 10].

\section{B. The Plaquette Basis}

For an isotropic $\left(J_{\|}=J_{\perp} \equiv 1\right)$ two-leg ladder, an efficient decomposition can be carried out in terms of distinct pairs of adjacent rungs; these are the plaquettes. In such a decomposition, a $2 \times 4$ ladder, for example, would be viewed as a pair of interacting plaquettes. The ladder Hamiltonian can be written as the sum of $H_{0}$, which contains the intra-plaquette interactions, and $V$, which includes the interactions between plaquettes. The intra-plaquette Hamiltonian $H_{0}$ is expressible as (see Fig. 1)

$$
H_{0}=\sum_{p=1}^{N_{P}} h_{0}^{(p)} ; \quad h_{0}^{(p)}=\left[\mathbf{S}_{1}^{(p)}+\mathbf{S}_{4}^{(p)}\right] \cdot\left[\mathbf{S}_{2}^{(p)}+\mathbf{S}_{3}^{(p)}\right]
$$


where $p$ labels a specific plaquette and the sum runs over all $N_{P}$ plaquettes in the ladder. As was shown in Ref. [5], $H_{0}$ is diagonal in the plaquette basis. The single-plaquette wavefunctions and energies are of the form:

$$
\begin{gathered}
\left|\phi_{\alpha}\right\rangle=\left|\left(s_{1} s_{4}\right) \ell_{14},\left(s_{2} s_{3}\right) \ell_{23} ; j m\right\rangle, \\
\epsilon_{\alpha}=\frac{1}{2}\left[j(j+1)-\ell_{14}\left(\ell_{14}+1\right)-\ell_{23}\left(\ell_{23}+1\right)\right],
\end{gathered}
$$

where $\alpha \equiv\left\{\ell_{14}, \ell_{23}, j, m\right\}$ denotes a generic quantum label. In the plaquette basis the two diagonal pairs of spins are coupled to well-defined total angular momenta, $\ell_{14}$ and $\ell_{23}$, which can equal zero or one. These two link angular momenta are in turn coupled to a total plaquette angular momentum $j$ with projection $m$. In this scheme, the interaction $V$ is given by

$$
V=\sum_{p=1}^{N_{P}}\left[\mathbf{S}_{3}^{(p)} \cdot \mathbf{S}_{1}^{(p+1)}+\mathbf{S}_{4}^{(p)} \cdot \mathbf{S}_{2}^{(p+1)}\right] .
$$

Matrix elements of $V$ in the plaquette basis are computed in terms of five Racah coefficients, as was shown in Ref [5].

\section{Rayleigh-Schrödinger Perturbation Theory}

In this section we review some well-known results in perturbation theory [11, 12]. To formulate Rayleigh-Schrödinger perturbation theory for finite ladders, we let the Hamiltonian $H \rightarrow H(\lambda) \equiv H_{0}+\lambda V$ and make a consistent expansion of the energy and wavefunction in powers of the "small" parameter $\lambda$. That is, the exact wavefunction of $H(\lambda)$ is expanded in a power series in terms of the eigenstates of $H_{0}$

$$
\left|\Psi_{\alpha}\right\rangle=\sum_{n=0}^{\infty} \lambda^{n} \sum_{\beta} A_{\alpha \beta}^{(n)}\left|\Phi_{\beta}\right\rangle
$$

while the energy is expanded as

$$
E_{\alpha}=\sum_{n=0}^{\infty} \lambda^{n} E_{\alpha}^{(n)}
$$

Note that $A_{\alpha \alpha}^{(n)}=\delta_{n 0}, A_{\alpha \beta}^{(0)}=\delta_{\alpha \beta}$, and $E_{\alpha}^{(0)} \equiv \epsilon_{\alpha}$ is an eigenvalue of the unperturbed Hamiltonian $H_{0}$. It is straightforward to derive a recursion relation for the above coefficients which is well-suited for implementation on a computer to arbitrary order in perturbation theory. We obtain

$$
\begin{aligned}
E_{\alpha}^{(n)} & =\sum_{\beta}\left\langle\Phi_{\alpha}|V| \Phi_{\beta}\right\rangle A_{\alpha \beta}^{(n-1)}, \\
A_{\alpha \beta}^{(n)} & =\frac{1}{\epsilon_{\alpha}-\epsilon_{\beta}}\left[\sum_{\sigma} A_{\alpha \sigma}^{(n-1)}\left\langle\Phi_{\sigma}|V| \Phi_{\beta}\right\rangle-\sum_{m=1}^{n-1} E_{\alpha}^{(n-m)} A_{\alpha \beta}^{(m)}\right] .
\end{aligned}
$$

These formulas are very simple to apply to the ground state of a $2 \times N_{R}$ ladder consisting of $N_{P}=N_{R} / 2$ plaquettes. The unperturbed state is given by 


$$
\left|\Phi_{J=0}\right\rangle=\left|\phi_{0}\right\rangle \otimes\left|\phi_{0}\right\rangle \ldots\left|\phi_{0}\right\rangle .
$$

In the rung basis $\left|\phi_{0}\right\rangle$ represents the singlet state, while in the plaquette basis it is the $\left|\ell_{14}=\ell_{23}=1, j=0\right\rangle$ single-plaquette wavefunction. The situation becomes slightly more complicated for the $(J=1)$ one-magnon states. At zeroth order this state is $N$-fold degenerate (with $N=N_{R}$ or $N=N_{P}$ ) so Eq. 12 is no longer applicable. However, if we assume periodic boundary conditions, the total linear momentum becomes a good quantum number and serves to distinguish among the $N$ degenerate states, which we now write as:

$$
\left|\Phi_{J=1}^{(\nu)}\right\rangle=\frac{1}{\sqrt{N}} \sum_{j=1}^{N} \exp \left[i \frac{2 \nu \pi}{N} j\right]\left|\Phi_{J=1}^{(j)}\right\rangle,
$$

where the linear-momentum label runs from $\nu=0, \ldots, N-1$ and

$$
\left|\Phi_{J=1}^{(j)}\right\rangle=\left|\phi_{0}\right\rangle \otimes\left|\phi_{0}\right\rangle \ldots\left|\phi_{1}\right\rangle \ldots\left|\phi_{0}\right\rangle .
$$

Now $\left|\phi_{1}\right\rangle$ is the triplet state for the $j$ th rung, or the $\left|\ell_{14}=\ell_{23}=j=1\right\rangle$ single-plaquette wave function for the $j$ th plaquette. In this way, Eq. 12 can be used for each individual state in the degenerate band, as states with different linear momenta cannot mix at any order in perturbation theory. We have systematically determined that, for sufficiently small values of $\lambda$, our perturbative calculations agree essentially perfectly at large order with exact results obtained by direct diagonalization or Lanczos techniques.

\section{PERTURBATION THEORY IN COUPLED BASES: BULK SYSTEMS}

The essential concept that we use to extract perturbative quantities in the bulk limit from calculations for finite systems is that of "connectedness". It is well known in manybody theory (see, e.g., Refs. [0,11],13,[14]) that any given perturbative contribution to the coefficients $\left(E_{J=0}^{(n)}\right)$ appearing in the expansion for the ground-state energy [see Eqs. (11, 12)] consists only of spatially connected terms. This is also the basis of the connected-graph expansions alluded to in the Introduction. As is well-known [11, 13, 14], both connected and disconnected terms appear in Rayleigh-Schrödinger perturbation theory. However, the disconnected terms are canceled by contributions included in the subtractions appearing in the evaluation of the $A_{\alpha \beta}^{(n)}$, as shown in Eq. (12). The linked-cluster expansions were, of course, developed to avoid dealing with disconnected terms which would eventually be canceled anyway, the price being much greater conceptual complexity.

In this work, we employ Rayleigh-Schrödinger perturbation theory and find that the computational penalty for not excluding disconnected contributions from the outset is insignificant compared to the advantages arising form the simplicity of the formulation. Still, we must exploit the connectedness of the surviving contributions to extract bulk properties. In the present context, connected terms are those for which a single, finite cluster of adjacent plaquettes can be identified such that: 1) all interactions involve plaquettes within that cluster and 2) all plaquettes in the cluster are involved in one or more interactions (note that in the present discussion we make explicit reference to plaquettes; however, all the arguments remain valid in the rung basis, unless stated otherwise). It is apparent that, at order $n$ in perturbation theory, the largest such cluster contains $n+1$ plaquettes. Hence, we 
may conclude that, at $n$th order, no new kinds of clusters appear as we change the number of plaquettes, $N$, provided $N \geq n+1$. Instead, only the number of times that each type of cluster appears changes with $N$. As the energy must scale at most with $N$, we may conclude that the ground-state energy is generally expressible as:

$$
E_{J=0}^{(n)}(N)=A_{0}+B_{0} N
$$

where $A_{0}$ and $B_{0}$ are "intensive" coefficients, which are independent of $N$, for $N \geq n+1$. This simple, physically reasonable behavior-and more specifically the absence of terms depending on $N^{2}, N^{3}, \ldots$, is again related to connectedness [11,14. Indeed, it was in order to eliminate terms with "anomalous" $N$-dependence that the linked-cluster expansion was first proposed.

It is clear that, for the case of periodic boundary conditions, we may set $A_{0}=0$. Thus, to $n$th order in perturbation theory, the ground-state energy-per-site of an $N$-plaquette ladder becomes independent of the number of plaquettes for $N \geq n+1$. Obviously then, the $n$th order energy-per-site in the bulk limit can be expressed as:

$$
E_{J=0}^{(n)} / \text { site }=\frac{1}{4} E_{J=0}^{(n)}(N=n+1) /(n+1),
$$

where $E_{J=0}^{(n)}(N)$ is the singlet ground state for an $N$-plaquette ladder, assuming periodic boundary conditions.

The structure of the unperturbed one-magnon states $\left(\left|\Phi_{J=1}^{(\nu)}\right\rangle\right)$ or, equivalently, the fact that the gap is an intensive quantity, ensures that, in the bulk limit, the energy-per-site equals that of the ground state. Hence, we may conclude that all one-magnon states have the same extensive contribution as the ground state. Therefore,

$$
E_{J=1}^{(n)}(\nu, N)=A_{1}^{(\nu)}+B_{0} N
$$

Again, to $n$th order, $A_{1}^{(\nu)}$ can be determined from studying a finite ladder with at least $N=n+1$ plaquettes. For $\nu=0, A_{1}^{(\nu=0)}$ is just the spin gap at order $n$ and its value in the bulk limit is found simply by taking the difference between the $n$th order energies for the lowest one-magnon state and the ground state where both are evaluated for an $N=(n+1)$ plaquette system with periodic boundary conditions:

$$
\Delta^{(n)}=E_{J=1}^{(n)}(\nu=0, N=n+1)-E_{J=0}^{(n)}(N=n+1) .
$$

We use this formula to determine the perturbative contributions to the spin gap in the bulk limit presented in the following sections. Note that in the rung basis, it is the linear momentum corresponding to $\nu=N / 2$ (i.e., $k=\pi$ ) which generates the lowest one-magnon state. In this case one must employ a finite ladder containing an even number of rungs.

It is also easy to show that the $n$th order one-magnon dispersion relation has the following form:

$$
\Delta^{(n)}(k)=\sum_{x=0}^{n} \Delta_{x}^{(n)} \cos (k x)
$$

where $0 \leq k \leq 2 \pi$ is the linear momentum in lattice units. Note that that in the plaquette basis the gap is given by $\Delta^{(n)}=\Delta^{(n)}(k=0)$ while, in the rung basis, $\Delta^{(n)}=\Delta^{(n)}(k=\pi)$. It 
might be anticipated that, at order $n$, the $n+1$ coefficients which specify the full dispersion relation at this order could be extracted directly from the values of the differences

$$
\Delta^{(n)}(k=2 \nu \pi / N)=E_{J=1}^{(n)}(\nu, N=n+1)-E_{J=0}^{(n)}(N=n+1) .
$$

However, a moment's thought reveals that some of these one-magnon states are degenerate and, thus, energies for systems larger than $N=n+1$ are typically required. Indeed, for a $2 \times 8$ (eight-rung) system, a perturbative calculation in the rung basis determines the bulk values of the energy-per-site and the gap up to 7th-order in perturbation theory, but only to 4th-order for the dispersion relation. Nevertheless, it is always possible to ascertain the width of the one-magnon band at $n$-th order from calculations for systems with $N=n+1$ plaquettes, provided $N$ is even.

Finally, it should be noted that the structure of the ground state in the plaquette basis (and, indeed, in any basis in which the unperturbed ground state is made out of singlets) affords a less obvious but more efficient means of extracting the coefficients that determine the ground-state energy in the bulk limit. To see this, we first note that $\left|\Phi_{J=0}\right\rangle$ contains only $j=0$ plaquettes [see Eq. (13)]. Since the inter-plaquette interaction $V$, specified in Eq. (9), consists of rank-1 operators in the space of a single plaquette, any plaquette appearing in a connected cluster must experience at least two interactions in order that it be returned to its original $j=0$ state. For this reason, it can be shown that $E_{J=0}^{(n)}(N)$, when computed with open boundary conditions, may be expressed as:

$$
E_{J=0}^{(n)}(N)=\sum_{m=1}^{[n / 2]}(N-m) W_{m}^{(n)}(N=m+1),
$$

where $[n / 2]$ is the largest integer less than or equal to $n / 2$. Then, for example, the quantities $W_{1}^{(5)}$ and $W_{2}^{(5)}$ can be extracted from fifth-order calculations for systems with only two and three plaquettes. (Note that, in contrast, the extraction of fifth-order coefficients $\left(E_{J=0}^{(n)}\right)$ for the ground-state energy using periodic-boundary conditions and Eq. (17) requires calculations for systems with six plaquettes.) Then, the $n$ th-order energy-per-site in the bulk limit is easily found to be

$$
E_{J=0}^{(n)} / \text { site }=\frac{1}{4} \sum_{m=1}^{[n / 2]} W_{m}^{(n)}(N=m+1) .
$$

We use this formula to determine the bulk perturbative ground state energy contributions presented in the following sections.

\section{RESULTS}

\section{A. The Rung Basis}

We first demonstrate our method for extracting perturbative energies in the bulk limit from Rayleigh-Schrödinger calculations for finite systems. Fig. 2 shows rung-basis calculations for the ground state energy and for the spin gap for systems with 4, 6, and 8 rungs. These calculations were done for $\lambda=0.25$ so convergence to DMRG values [15] is very fast. 
Fig. 2 shows that, at orders $n=2$ and 3, all calculations give the same per-rung energy and spin-gap contributions, as we would expect from the arguments of the preceding section. At $n=4$, the 6 - and 8-rung values coincide, but now differ from the 4-rung results, again consistent with our expectations. Finally, the 6- and 8-rung values begin to differ for $n \geq 6$. Note that our 7th-order perturbative result for the spin gap agrees with the DMRG value to about $0.07 \%$ (for the energy per site both results are indistinguishable to five significant figures). The left-hand side of Fig. 2 also demonstrates that, when Eq. (23) is used, the contributions to the ground-state energy extracted from 4-rung calculations with open boundary conditions agree with those obtained from an 8-rung ladder with periodic boundary conditions through $n=7$, as expected. This result embodies the power of the method, and the high degree of symmetry of the unperturbed ground state and the residual interaction. Indeed, an 8-rung calculation affords the computation of the ground-state energy - up to 15 th order in perturbation theory.

Moreover, from one such calculation, namely, a $2 \times 8$ ladder at a single value of $\lambda$, one can extract the perturbative coefficients for the ground-state energy and the spin gap up to 7th-order. That is, the ground-state energy per site is given by

$$
E_{0} / N=-\frac{3}{8}-\frac{3}{16} \lambda^{2}-\frac{3}{32} \lambda^{3}+\frac{3}{256} \lambda^{4}+\frac{45}{512} \lambda^{5}+\frac{159}{2048} \lambda^{6}-\frac{55}{2048} \lambda^{7},
$$

while for the spin gap we obtain:

$$
\Delta=1-\lambda+\frac{1}{2} \lambda^{2}+\frac{1}{4} \lambda^{3}-\frac{1}{8} \lambda^{4}-\frac{35}{128} \lambda^{5}-\frac{157}{1024} \lambda^{6}+\frac{503}{2048} \lambda^{7} .
$$

Of course, these two expressions can be used for arbitrary values of $\lambda$, provided these values lie within the radius of convergence of the perturbative expansions (more on this point later). In Fig. 3 we have displayed the bulk values for the ground-state energy per site and the spin gap to various orders in perturbation theory; we have also included exact results obtained on a $2 \times 8$ ladder. We observe that in the strong-coupling regime $(\lambda \lesssim 0.5)$ the convergence of our results is very good. This should not come as a surprise, as the rung basis has been customized to handle the strong-coupling region. In contrast, the convergence of the perturbative results in the intermediate- to isotropic-coupling region $(\lambda \gtrsim 0.8)$ is harder to assess. However, the fact that there is no systematic improvement - nor convergence - in the results as one goes to higher orders in perturbation theory, strongly suggests that the expansion might no longer be convergent.

In an effort to establish quantitatively the radius of convergence of the perturbative expansion, we have compared high-order perturbative and exact results for finite ladders over a range of $\lambda$ values. For example, Fig. 1 shows the ground-state energy per site through 50th order in perturbation theory for 4-, 6- and 8-rung ladders as a function of $\lambda$. The radius of convergence is determined by the onset of wild excursions in these quantities at $\lambda_{c} \simeq 0.8$. These perturbative results are indistinguishable from the Lanczos energies for $\lambda \leq \lambda_{c}$. As the value of $\lambda_{c}$ appears to have very little dependence on the size of the ladder, we assume it is also appropriate to the bulk system. Thus, we conclude that the radius of convergence of the perturbative expansion in the rung basis is $\lambda_{c}=0.8$. This result indicates that perturbative expansions in the rung basis are unsuitable for dealing with the physically-interesting case of isotropic $(\lambda=1)$ ladders.

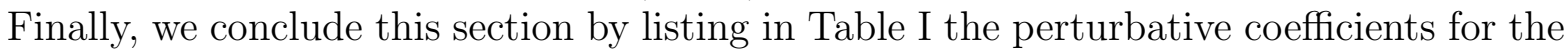
one-magnon dispersion relation, as given in Eq. (20). 


\section{B. The Plaquette Basis}

Plaquette-based perturbative results for the ground-state energy per site and for the spin gap appear in Table [I]. By carrying out Rayleigh-Schrödinger calculations for isotropic $\left(J_{\|}=J_{\perp}\right)$ ladders as large as $2 \times 12$ (i.e., six plaquettes), we have been able to determine the ground-state energy per site through 11th order and the spin gap through fourth order. Through 11th order, the ground-state energy is within $0.02 \%$ of the DMRG [2] value while at fourth order, the perturbative estimate for the spin gap agrees with the DMRG value to better than 10\%. The perturbative energy per site is plotted versus the order of perturbation in Fig. 5 where the rapid convergence to the DMRG value is apparent. A similar plot for the spin gap is shown in Fig. 6. Here convergence is less dramatic presumably due to the relatively low order of perturbation theory to which we are restricted. As became apparent in the previous section, there is no point in presenting a similar table or figures for rung-basis perturbation theory, as it does not converge for $\lambda=1$ which is the only point where direct rung versus plaquette comparisons are possible.

To extract the radius of convergence in plaquette-based perturbation theory we have again compared high-order perturbative and exact results for finite ladders over a range of $\lambda$ values. Indeed, Fig. 7 shows the ground-state energy per site through 50th order in perturbation theory for 4-, 5- and 6-plaquette systems as a function of $\lambda$. Notice that for the present case the onset of the wild excursions is now at $\lambda_{c} \simeq 1.4$; once more the agreement with exact calculations using direct diagonalization or Lanczos methods is essentially perfect for $\lambda \leq \lambda_{c}$. Fig. 8 shows similar plots for the lowest triplet state of ladders with 4 and 5 plaquettes. On the basis of the behavior shown, we estimate that $\lambda_{c} \simeq 1.25$ for this state. It is therefore straightforward to conclude that the plaquette basis offers, to date, the only reliable perturbative approach for the treatment of the physically interesting [8] case of isotropic $(\lambda=1)$ spin ladders.

Finally we conclude this section by presenting in Fig. 9 the perturbative one-magnon dispersion relation for the plaquette basis. The curves presented in these figures were generated by inserting the coefficients appearing in Table [II into Eq. (20).

\section{SUMMARY AND CONCLUSIONS}

We have employed rung and plaquette bases to study bulk properties of Heisenberg spin-1/2 ladders using Rayleigh-Schrödinger perturbation theory. In both cases, the mere selection of an angular momentum coupled basis enables one to separate the Hamiltonian into a unperturbed part, which is solved exactly, and a "residual interaction" which is treated in perturbation theory. In this way the starting point for the calculation are unperturbed wavefunctions consisting of a direct product of spin singlets for the ground state, and a degenerate "one-magnon" band in which one spin singlet has been turned into a triplet. The unperturbed ground-state energies per site in the rung and plaquette bases are given by $-3 / 8$ and $-1 / 2$, respectively; the spin gap starts at $\Delta=1$ in both cases.

The one-dimensional nature of the problem enabled us to employ powerful methods for the extraction of high order perturbative coefficients for the bulk system from calculations on relatively small ladders. Indeed, using very general arguments - related to the linked-cluster expansion and to the scaling of observables in the bulk limit-we have shown that from a 
calculation on a ladder with $N$ clusters (a cluster being a rung or a plaquette) we can extract all perturbative coefficients for the ground-state energy and for the spin gap up to order $N-1$. Moreover, with a simple modification, namely using open-boundary conditions, the groundstate energy per site could be computed up to order $2 N-1$. Yet, because of degeneracies in the one-magnon band, perturbative coefficients for the one-magnon dispersion relation could only be extracted up to order $N-2$ by combining results from Rayleigh-Schrödinger calculations for ladders with $N$ and $N-1$ plaquettes.

For the rung basis we have carried out calculations on a relatively small $2 \times 8$ ladder; this sort of computations can now be carried out routinely on a personal computer. Yet, the power of the method allowed us to evaluate the ground-state energy and the spin gap to 7th-order in perturbation theory. Perhaps as important, we could reliably estimate the radius of convergence of the perturbative expansion in the rung basis. This is essential, as such expansions are routinely assumed to be meaningful in the isotropic $\left(J_{\|} / J_{\perp} \sim 1\right)$ limit [1, [,, 4 . We have found a radius of convergence for the perturbative expansion in the rung basis of only $\lambda=J_{\|} / J_{\perp} \simeq 0.8$.

Similar calculations were also carried out in the plaquette basis. Here the parameter $\lambda$ scales the inter-plaquette to the intra-plaquette interactions; the isotropic $\left(J_{\|}=J_{\perp}\right)$ limit was obtained by setting $\lambda=1$. We were able to perform calculations for the singlet ground state on a $2 \times 12$ ladder. For the triplet states, we were restricted to a $2 \times 10$ ladder. Even when a ladder of such large size was employed, the spin gap could only be computed up to 4th-order in perturbation theory; the ground-state energy was computed with openboundary conditions so 11th-order results were reported. At first sight this appears as a drawback relative to the rung-basis calculations described earlier. However, on close examination one observes that rung-basis perturbation expansions in the physically-interesting case of the isotropic limit $(\lambda=1)$ are not feasible even in principle. In contrast, the isotropic point is well within the radius of convergence - estimated to be at $\lambda_{c} \simeq 1.25$-for plaquettebased expansions. Finally, by a combination of 4- and 5-plaquette results, we were able to extract the one-magnon band dispersion relation through 3rd order in perturbation theory.

In conclusion, we have formulated perturbation theory for two-leg Heisenberg spin ladders in two angular momentum coupled bases, namely the "rung" and "plaquette" bases. By exploiting the connectedness of perturbative energy contributions, we have been able to straightforwardly extract quantities appropriate to the bulk system from RayleighSchrödinger perturbation theory for finite ladders. We find that the radius of convergence in the rung basis is $\lambda_{c} \simeq 0.8$ while, in the plaquette basis, it is $\lambda_{c} \simeq 1.25$. Clearly, the latter basis is strongly preferred when treating the physically interesting case of isotropic spin ladders which corresponds to $\lambda_{c}=1$ in both bases. As we have stressed elsewhere [0.6], the plaquette basis is especially well-suited to the study of many aspects of spin ladders. Its success in the context of perturbation theory as presented here encourages us to use it in other investigations including a mean-field treatment of ladders (see, e.g., Ref. [G] for a rung-basis approach) and in a $t-J$ model aimed at the very interesting question of the dynamics of doped ladders (again, see, e.g., Ref. [16] for a rung-basis approach). These studies will be the subject of future reports. 


\section{ACKNOWLEDGMENTS}

We thank Martin Gelfand for useful discussions and for providing us with his unpublished plaquette results obtained independently using different techniques. We also thank Markus Laukamp for making available the DMRG results. This work was supported by the DOE under Contracts Nos. DE-FC05-85ER250000, DE-FG05-92ER40750 and DE-FG0393ER40774. 


\section{REFERENCES}

[1] T. Barnes, E. Dagotto, J. Riera and E.S. Swanson, Phys. Rev. B47, 3196 (1993).

[2] S.R. White, R.M Noack and D.J. Scalapino, Phys. Rev. Lett. 73, 886 (1994).

[3] M. Reigrotzki, H. Tsunetsugu and T.M. Rice, J. Phys. C 6, 9235 (1994); cond-mat/9408023.

[4] Sudha Gopalan, T.M. Rice and M. Sigrist, Phys. Rev. B49, 8901 (1994); cond-mat/9312026.

[5] J. Piekarewicz and J.R. Shepard, Phys.Rev. B56, 5366 (1997); cond-mat/9612139.

[6] J. Piekarewicz and J.R. Shepard, Phys.Rev. B, in press; cond-mat/9707207.

[7] Martin P. Gelfand, Rajiv R.P. Singh, and David A. Huse, Journal of Stat. Phys. 59, 1093 (1990) and references contained therein.

[8] E. Dagotto and T.M. Rice, Science 271, 618 (1996).

[9] L.C. Biedenharn and J.D. Louck, Angular Momentum in Quantum Physics; Theory and Applications, Addison-Wesley (Reading) 1981.

[10] D.M. Brink and G.R. Satchler, Angular Momentum, Clarendon Press (Oxford) 1993.

[11] A. deShalit and H. Feshbach, Theoretical Nuclear Physics, Vol. 1, John Wiley and Sons (New York) 1974.

[12] Leonard I. Schiff, Quantum mechanics, McGraw-Hill (New York) 1968.

[13] A.L. Fetter and J.D. Walecka, Quantum Theory of Many-Particle Systems, McGraw-Hill (New York) 1971.

[14] J.W. Negele and H. Orland, Quantum Many-Particle Systems, Addison-Wesley (Redwood City) 1988.

[15] M. Laukamp, private communication.

[16] M. Sigrist, T.M. Rice and F.C. Zhang, Phys.Rev. B49, 12058 (1994); cond-mat/9401019. 


\section{FIGURES}
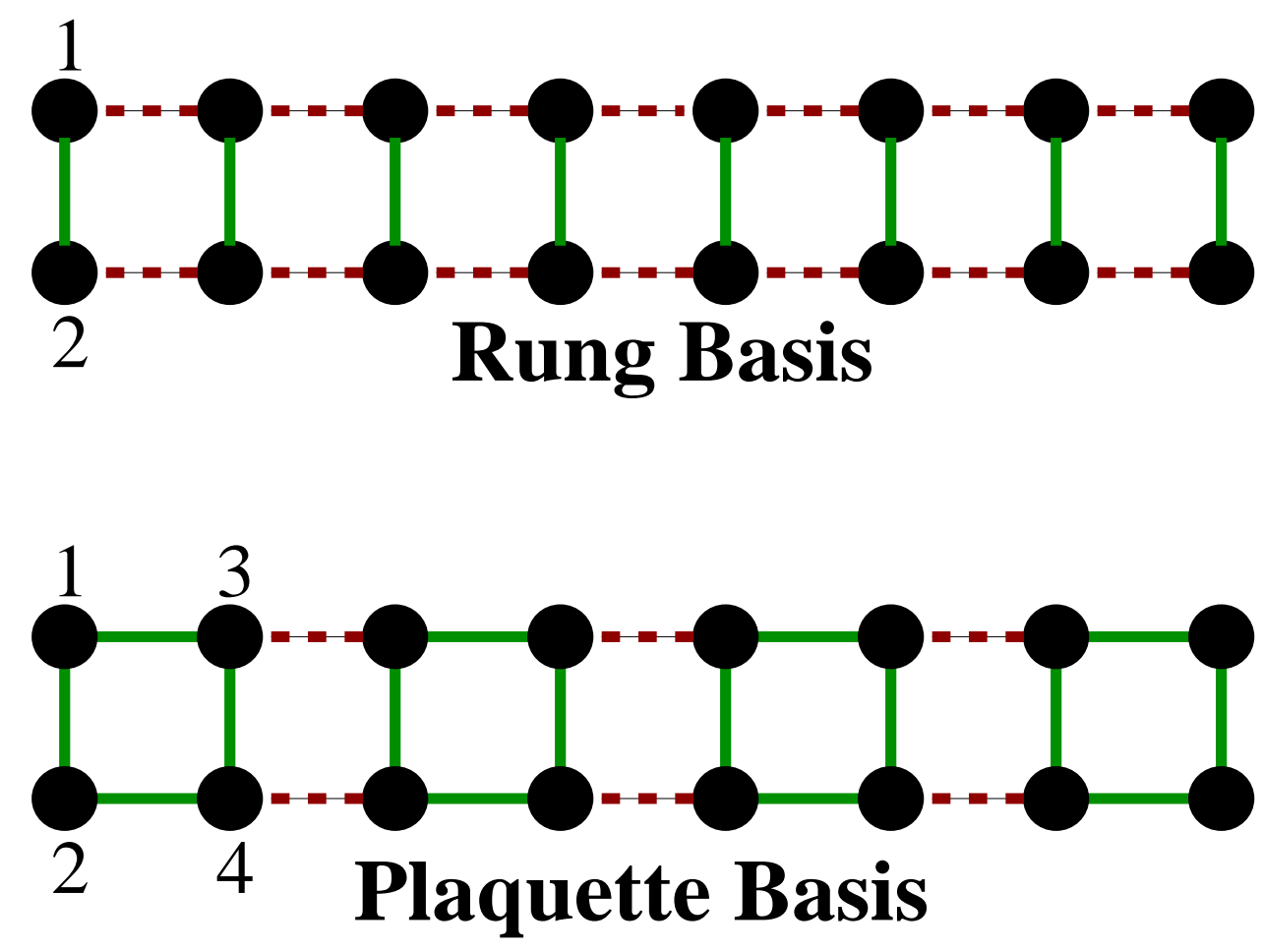

FIG. 1. Schematic representation of the rung and plaquette bases. The solid links represent those interactions which are diagonal in the basis; the dashed links represent the "perturbations". 

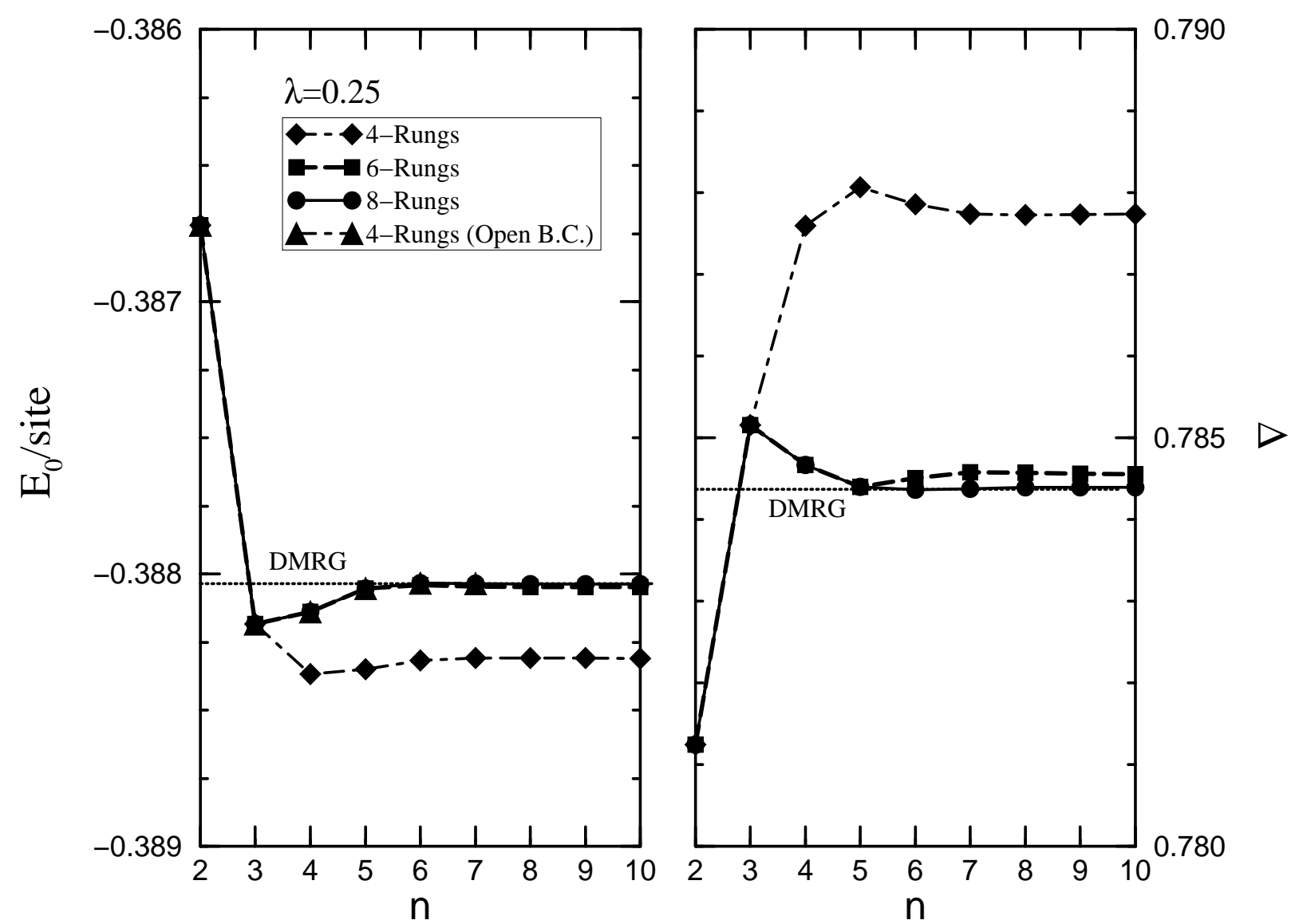

FIG. 2. Rung-basis perturbation theory results through order $n=10$ for the ground-state energy per site (left) and for the spin gap (right) for systems with 4, 6 and 8 rungs using periodic boundary conditions. For the ground-state energy, the triangles display a 4-rung calculation with open boundary conditions (see text for an explanation). Here $\lambda=0.25$ and the DMRG values are from Ref. [15] 

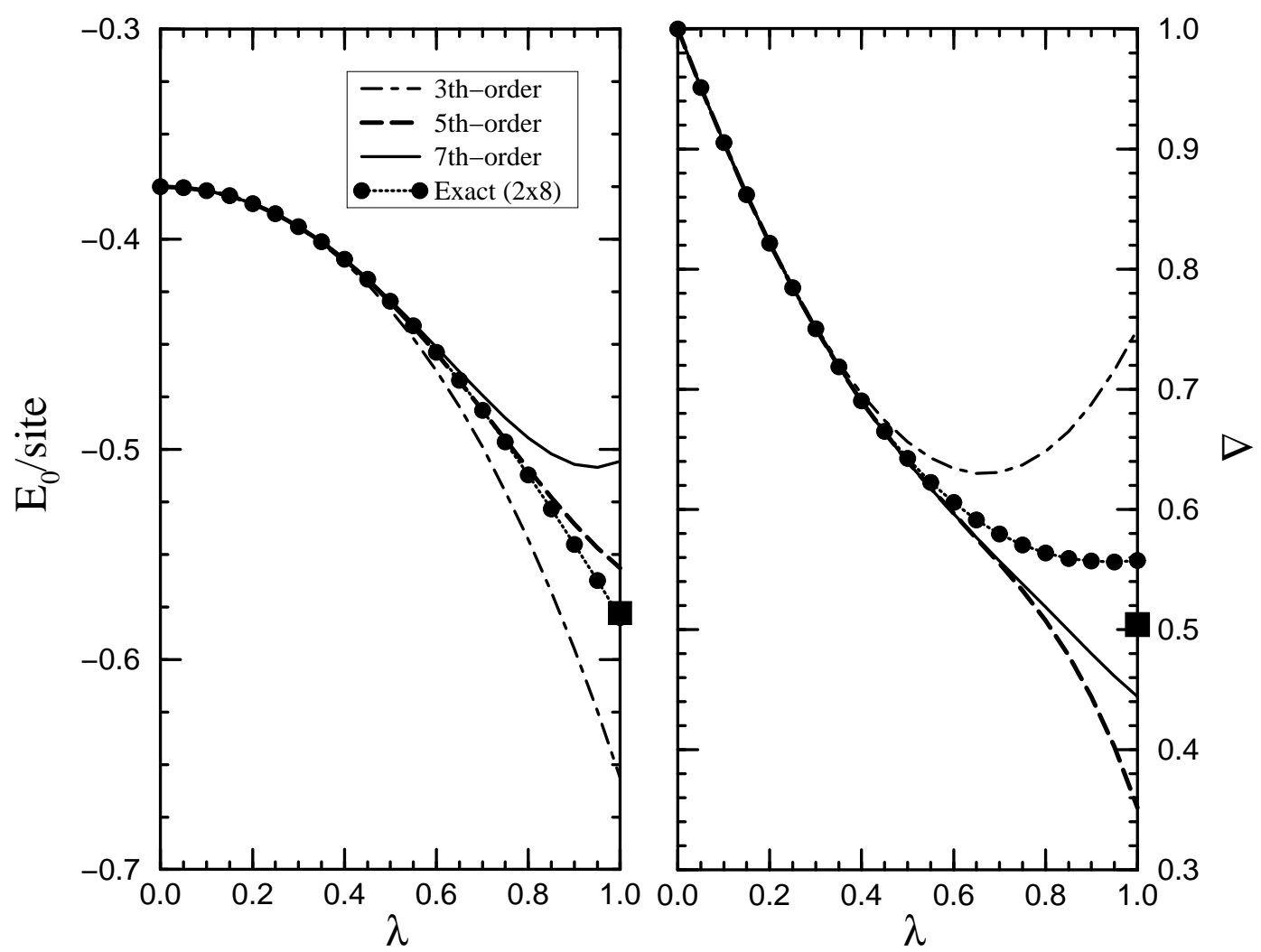

FIG. 3. Ground-state energy per site (left) and spin gap (right) in the bulk limit as a function of $\lambda$ in the rung basis. Various orders of perturbation theory are displayed as well as the exact results on an 8-rung ladder. The DMRG values [2] for the bulk system at the isotropic point $(\lambda=1)$ are displayed with a solid square. 


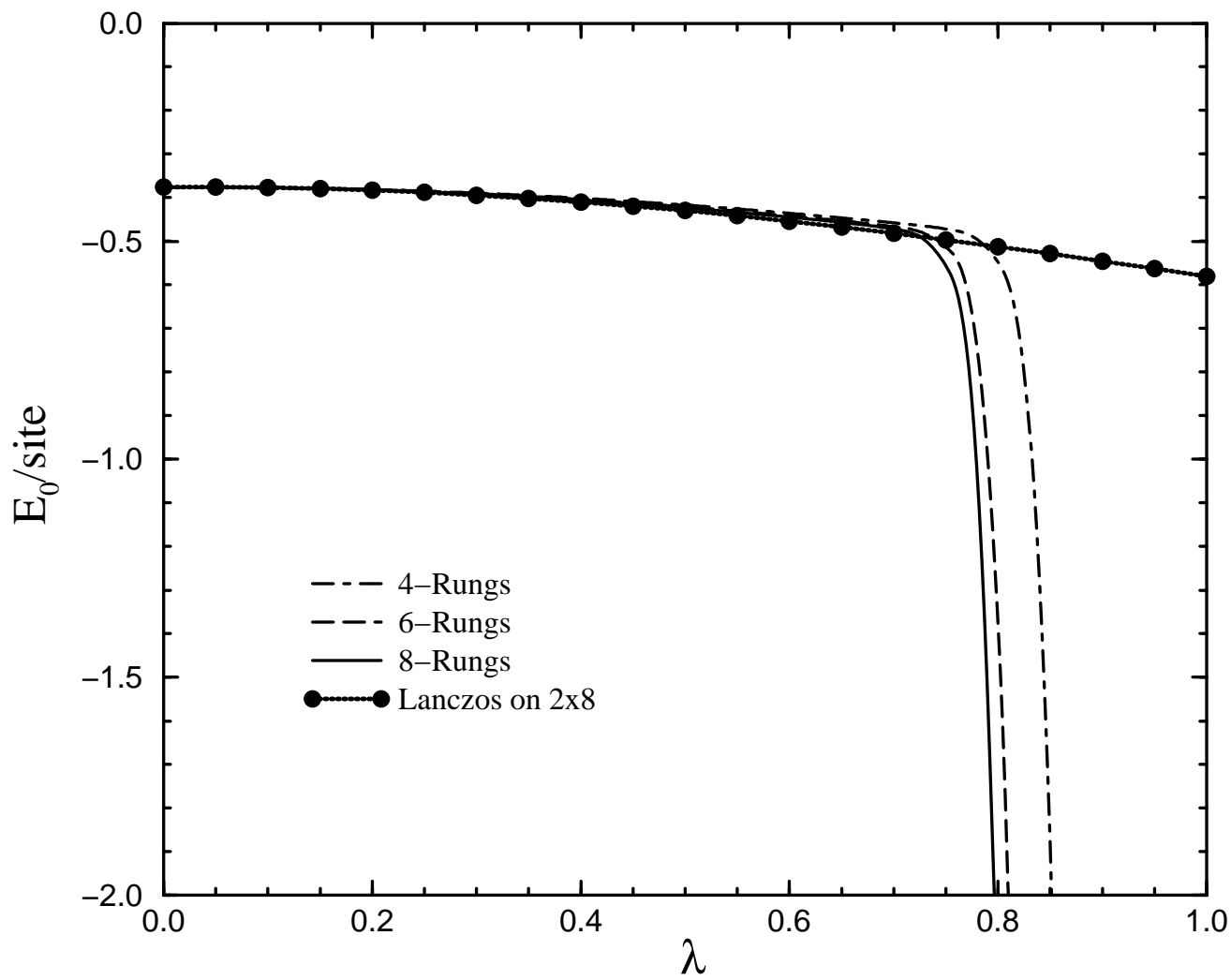

FIG. 4. Ground state energy per site for 4-, 6-, and 8-rung ladders at 50th order in perturbation theory as a function of $\lambda$. We conclude that the radius of convergence of the rung-basis perturbative expansion is about $\lambda_{c}=0.8$. 


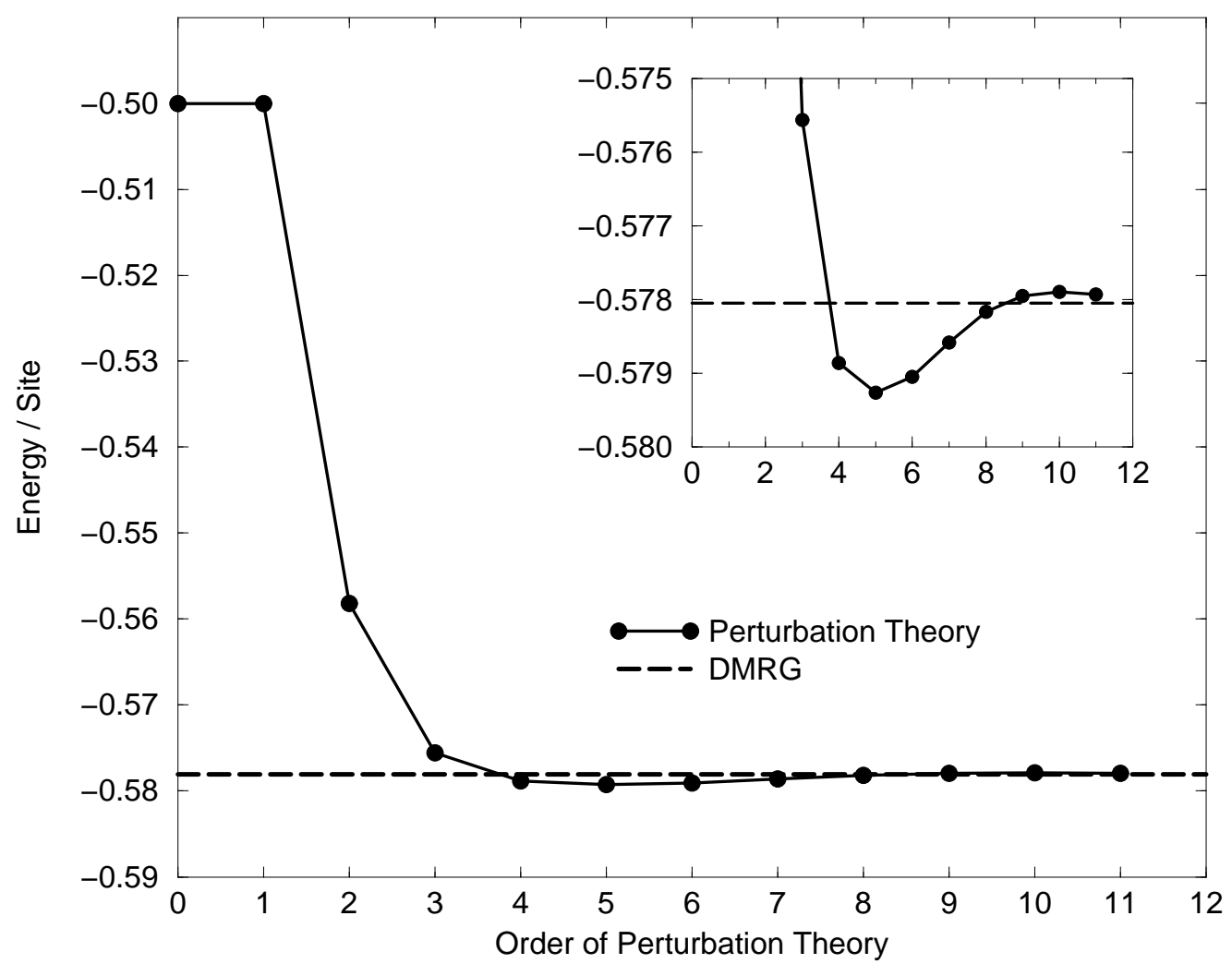

FIG. 5. Ground state energy per site in the bulk limit versus order of perturbation theory in the plaquette basis. The inset shows the same results but on an expanded scale. The DMRG value is from Ref. [2]. 


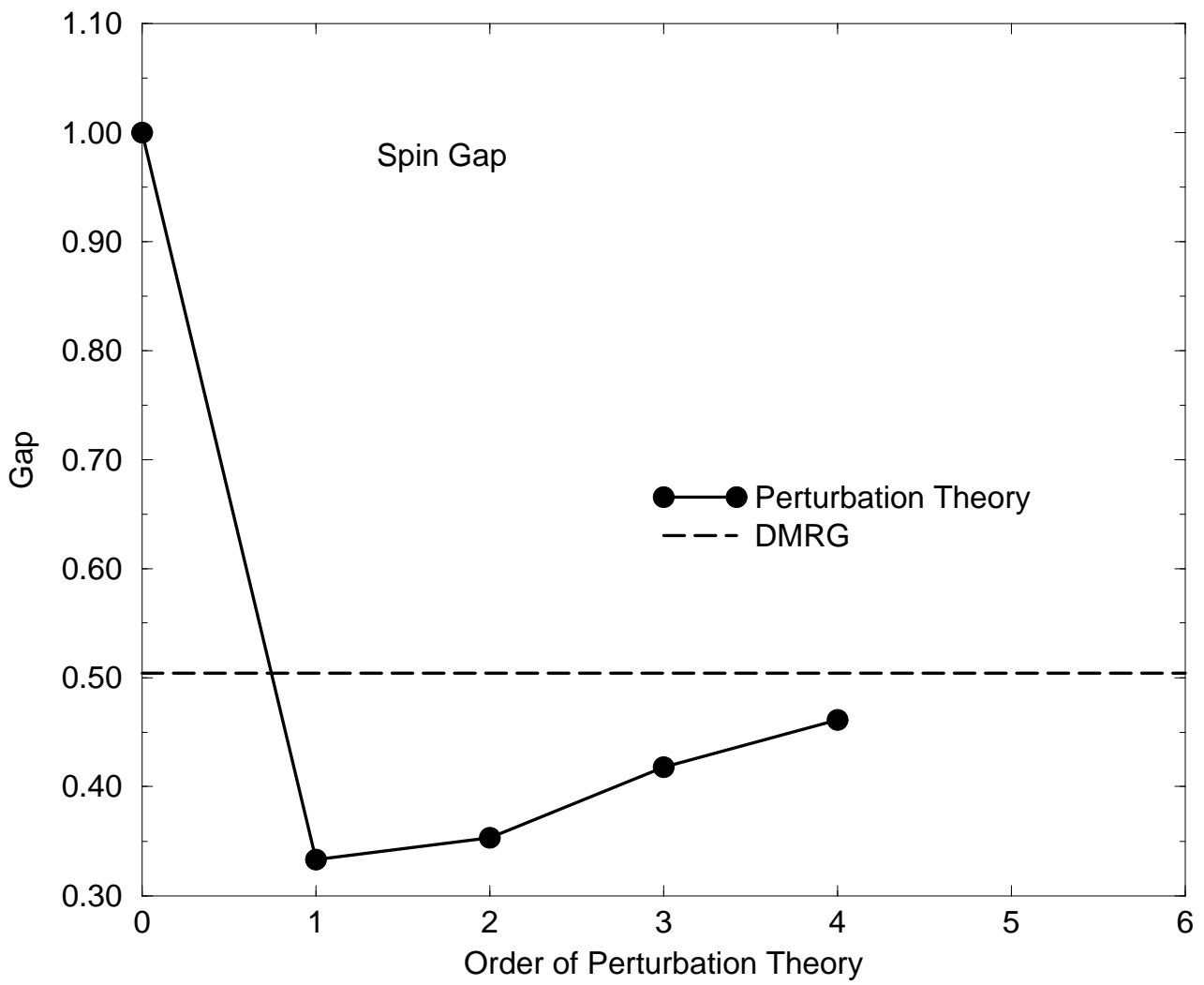

FIG. 6. Same as Fig. 5 but for the spin gap. 


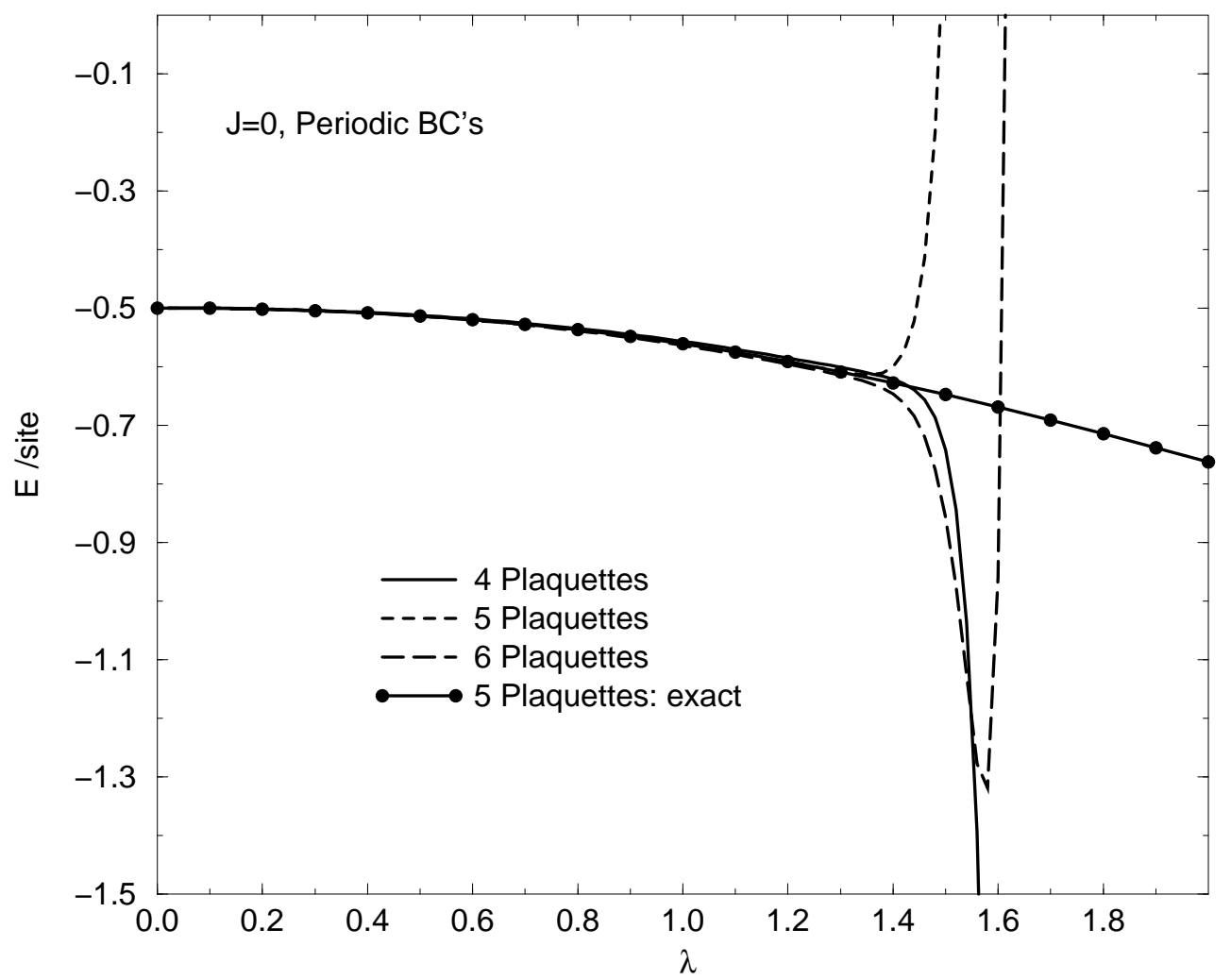

FIG. 7. Ground state energy per site for 4-, 5-, and 6-plaquette systems at 50th order in perturbation theory as a function of $\lambda$ which scales the inter-plaquette interaction (see the subsection on Rayleigh-Schrödinger Perturbation Theory). We conclude that the radius of convergence of the plaquette-basis perturbative expansion is about $\lambda_{c}=1.4$. 


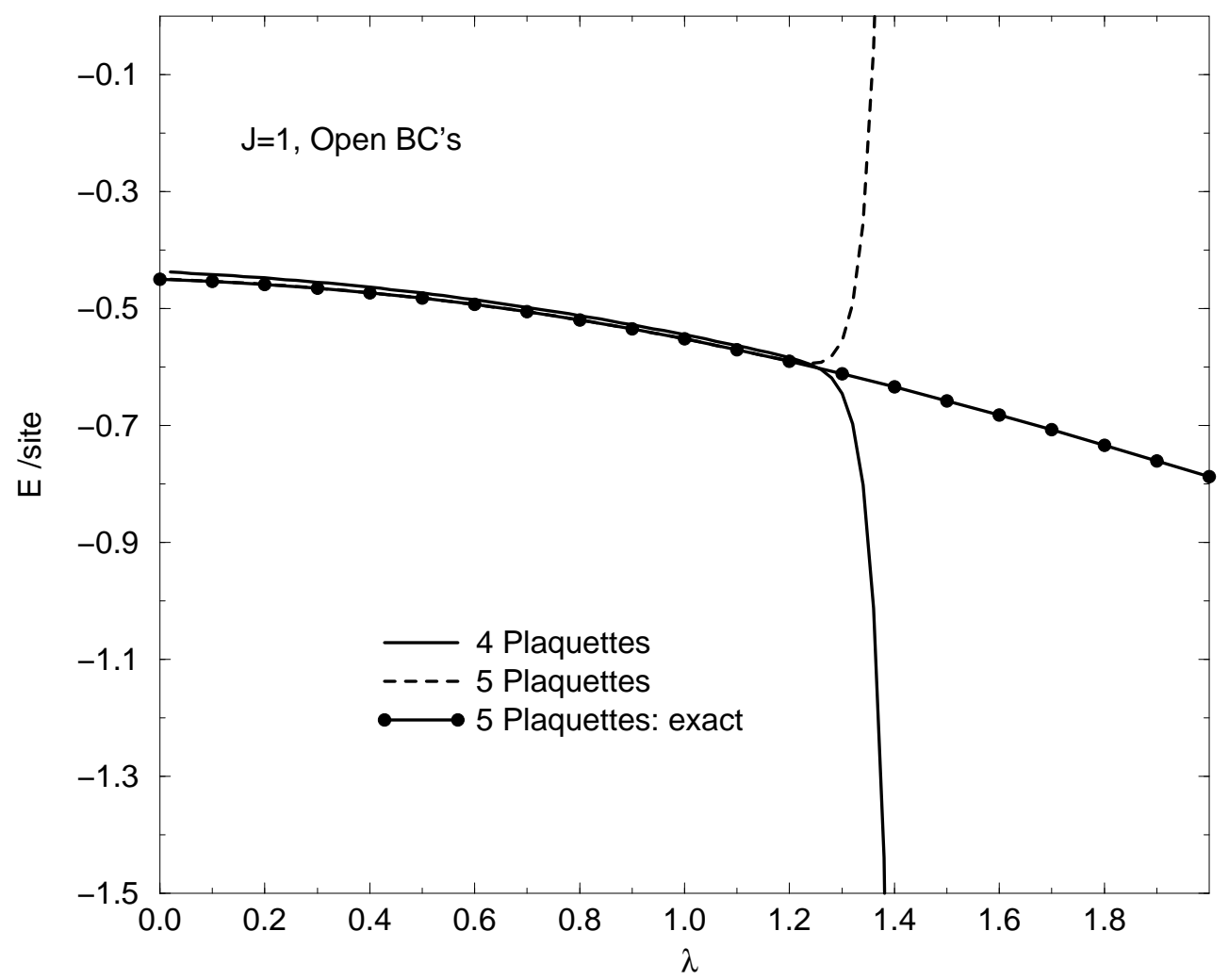

FIG. 8. Same as Fig. 7 but for the lowest triplet state. We conclude that the radius of convergence of the plaquette-basis perturbative expansion here is about $\lambda_{c}=1.25$. 


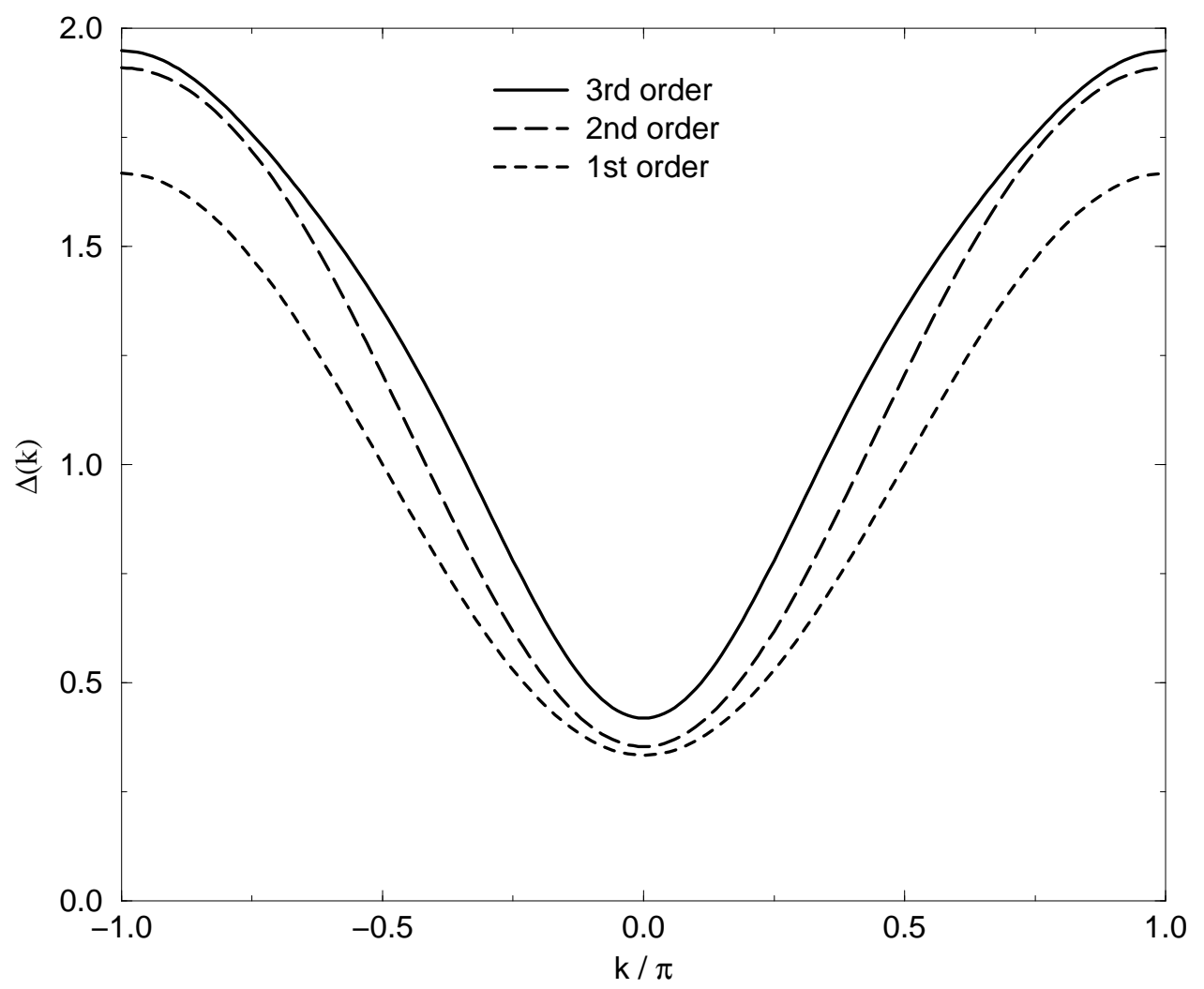

FIG. 9. One-magnon dispersion relation at various orders of plaquette-basis perturbation theory. 


\section{TABLES}

TABLE I. Rung-based perturbation theory for the one-magnon dispersion relation; see Eq. (20).

\begin{tabular}{cccccc}
\hline \hline Order $n$ & $\Delta_{0}^{(n)}$ & $\Delta_{1}^{(n)}$ & $\Delta_{2}^{(n)}$ & $\Delta_{3}^{(n)}$ & $\Delta_{4}^{(n)}$ \\
\hline 0 & 1 & & & & \\
1 & 0 & 1 & & & \\
2 & $3 / 4$ & 0 & $-1 / 4$ & & \\
3 & $3 / 8$ & $-1 / 4$ & $-1 / 4$ & $1 / 8$ & \\
4 & $-13 / 64$ & $-5 / 16$ & $-5 / 160$ & $1 / 8$ & $-5 / 64$ \\
\hline \hline
\end{tabular}

TABLE II. Plaquette-based perturbation theory for the bulk energy per site and spin gap of isotropic 2-leg spin ladders. [see Eq. (11)] DMRG results are from Ref. [2].

\begin{tabular}{ccc}
\hline \hline Order $n$ & $E_{0}^{(n)}$ & $\Delta^{(n)}$ \\
\hline 0 & -0.5 & 1 \\
1 & 0 & $-2 / 3$ \\
2 & -0.05815972 & 0.01967593 \\
3 & -0.01739728 & 0.06493538 \\
4 & -0.00329927 & 0.04306155 \\
5 & -0.00040692 & \\
6 & 0.00021569 & \\
7 & 0.00046854 & \\
8 & 0.00041483 & \\
9 & 0.00021860 & \\
10 & 0.00005215 & \\
11 & -0.00003610 & 0.46100619 \\
\hline Total & -0.57792948 & 0.504 \\
\hline DMRG & -0.57804314 & \\
\hline \hline
\end{tabular}

TABLE III. Plaquette-based perturbation theory for one-magnon dispersion relation; see Eq. (20).

\begin{tabular}{ccccc}
\hline \hline Order $n$ & $\Delta_{0}^{(n)}$ & $\Delta_{1}^{(n)}$ & $\Delta_{2}^{(n)}$ & $\Delta_{3}^{(n)}$ \\
\hline 0 & 1 & & & \\
1 & 0 & $-2 / 3$ & $-1 / 27$ & \\
2 & $145 / 864$ & $-1 / 9$ & -0.04822531 & $-1 / 27$ \\
3 & 0.10022425 & 0.04997348 & & \\
\hline \hline
\end{tabular}

